\title{
Correlation of the highest energy cosmic rays with nearby extragalactic objects
}

\author{
The Pierre Auger Collaboration* \\ Observatorio Pierre Auger, Avenida San Martín Norte 304, \\ (5613) Malargüe, Mendoza, Argentina
}

\begin{abstract}
Using data collected at the Pierre Auger Observatory during the past 3.7 years, we demonstrated a correlation between the arrival directions of cosmic rays with energy above $\sim 6 \times 10^{19}$ electron volts and the positions of active galactic nuclei (AGN) lying within $\sim 75$ megaparsecs. We rejected the hypothesis of an isotropic distribution of these cosmic rays with at least a $99 \%$ confidence level from a prescribed a priori test. The correlation we observed is compatible with the hypothesis that the highest energy particles originate from nearby extragalactic sources whose flux has not been substantially reduced by interaction with the cosmic background radiation. AGN or objects having a similar spatial distribution are possible sources.
\end{abstract}

Cosmic rays are energetic particles and nuclei from space that strike the Earth's atmosphere. Their energies vary from a few $10^{8} \mathrm{eV}$ to beyond $10^{20} \mathrm{eV}$. The flux of cosmic rays at Earth decreases very rapidly with energy, from a few particles per square centimeter per second in the low-energy region to less than one particle per square kilometer per century above $10^{20} \mathrm{eV}$. The identification of the sources of ultrahigh-energy cosmic rays (UHECR) with energies $\sim 10^{20} \mathrm{eV}$

${ }^{*}$ The full list of authors and their affiliations appears at the end of this paper. 
has been a great challenge since they were first observed in 1962 (1). Because cosmic rays at these energies are not expected to be confined by magnetic fields in the disk of our galaxy, and indeed no significant excess from the direction of the Milky Way has been observed, it is likely that they originate outside the Galaxy. Until now there has been no experimental confirmation of this hypothesis.

Because of their very low flux, UHECR can only be detected through their interaction with the Earth's atmosphere, producing a cascade of billions of particles that excite nitrogen molecules in the air along their path and spread over a large area when they reach the ground. The Pierre Auger Southern Observatory (2), now nearing completion in Argentina, was designed to simultaneously observe the shower particles at ground level and the associated fluorescence light generated in the atmosphere. A large array of 1600 surface detectors (SDs), laid out as an equilateral triangular grid with 1500-m spacing, covers an area of $3000 \mathrm{~km}^{2}$ and detects the particles at ground level by means of the Cherenkov radiation they produce in water. At each of four sites on the periphery of the instrumented area, six inward-facing optical telescopes observe the sky on clear moonless nights. These devices measure the atmospheric fluorescence light produced as an extensive air shower passes through the field of view. The two techniques - the SDs and the fluorescence detectors (FDs) - are complementary, and also provide cross-checks and redundancy in the measurement of air shower parameters. The SD measures the two-dimensional lateral structure of the shower at ground level, whereas the FD records the longitudinal profile of the shower during its development through the atmosphere. In Figure 1, we present the layout of the Observatory as of 30 September 2007.

The Pierre Auger Southern Observatory has been taking data stably since January 2004. The large exposure of its ground array, combined with accurate energy and arrival direction measurements, calibrated and verified from the hybrid operation with the fluorescence detectors, provides an opportunity to explore the spatial correlation between cosmic rays and their sources 
in the sky.

If cosmic rays with the highest energies are predominantly protons or nuclei, only sources closer than about $200 \mathrm{Mpc}$ from Earth can contribute appreciably to the observed flux above $60 \mathrm{EeV}\left(1 \mathrm{EeV}=10^{18} \mathrm{eV}\right)$. Protons or nuclei with energies above $60 \mathrm{EeV}$ interact with the cosmic microwave background $(3,4,5)$, leading to a strong attenuation of their flux from distant sources. This attenuation is known as the Greisen, Zatsepin and Kuzmin (GZK) effect, from the names of the three physicists that predicted it. If the sources of the most energetic cosmic rays are relatively nearby and are not uniformly distributed, then an anisotropic arrival distribution is expected, provided the particles have a sufficiently small charge and a sufficiently high energy for their directions to be minimally perturbed by intervening magnetic fields.

Anisotropy of the cosmic rays with the highest energies could manifest as clustering of events from individual point sources or through the correlation of arrival directions with a collection of astronomical objects. The Akeno Giant Air Shower Array (AGASA) Collaboration claimed some excess of clustering at small angular scales compared to isotropic expectations (6), but this was not supported by data recorded by the HiRes experiment (7). Analyses of data recorded by several air-shower experiments revealed a general correlation with the direction of the supergalactic plane $(8,9)$, where several nearby galaxies cluster, but with limited statistical significance.

AGN have long been considered sites where energetic-particle production might take place and where protons and heavier nuclei could be accelerated up to the highest energies yet measured $(10,11)$. Here, we report the observation of a correlation between the arrival directions of the cosmic rays with highest energies measured by the Pierre Auger Observatory and the positions of nearby AGN from the $12^{\text {th }}$ edition of the catalog of quasars and active nuclei by Véron-Cetty and Véron (V-C catalog) $(12)$. 
Data set and method The data set analyzed here consists of the cosmic-ray events recorded by the surface array of the Observatory from 1 January 2004 to 31 August 2007. It contains 81 events with reconstructed energies above $40 \mathrm{EeV}$ and zenith angles smaller than $60^{\circ}$. The integrated exposure is $9.0 \times 10^{3} \mathrm{~km}^{2}$ sr year.

We only use recorded events if they meet strict criteria with regard to the quality of the reconstruction of their energy and direction. The selection of those events is done via a quality trigger (13) which is only a function of the topology of the footprint of the event on the ground. This trigger requires that the detector with the highest signal must be surrounded by five active nearest neighbors, and that the reconstructed shower core be inside an active equilateral triangle of detectors. This represents an efficient quality cut while guaranteeing that no crucial information is missed for the shower reconstruction.

The arrival direction of a cosmic ray is a crucial ingredient in our study. The event direction is determined by a fit of the arrival times of the shower front at the SD. The precision achieved in the arrival direction depends on the clock resolution of each detector and on the fluctuations in the time of arrival of the first particle (14). The angular resolution is defined as the angular aperture around an arrival direction of cosmic rays within which $68 \%$ of the showers are reconstructed. This resolution has been verified experimentally with events for which two independent geometrical reconstructions can be performed. The first test uses hybrid events, which are measured simultaneously by the SD and the FD; the second one uses events falling in a special region of our array where two surface stations are laid in pairs $11 \mathrm{~m}$ apart at each position. Events that triggered at least six surface stations have energies above $10 \mathrm{EeV}$ and an angular resolution better than $1^{\circ}(15,16)$.

The energy of each event is determined in a two-step procedure. The shower size $S$, at a reference distance and zenith angle, is calculated from the signal detected in each surface station and then converted to energy with a linear calibration curve based on the fluorescence telescope 
measurements $(17)$. The uncertainty resulting from the adjustment of the shower size, the conversion to a reference angle, the fluctuation from shower to shower, and the calibration curve amounts to about $18 \%$. The absolute energy scale is given by the fluorescence measurements and has a systematic uncertainty of $22 \%(\sqrt{18})$. The largest systematic uncertainty arises primarily from an incomplete knowledge of the yield of photons from the fluorescence of atmospheric nitrogen (14\%), the telescope calibration $(9.5 \%)$ and the reconstruction procedure $(10 \%)$. Additional uncertainty in the energy scale for the set of high-energy events used in the present analysis is due to the relatively low statistics available for calibration in this energy range.

Events with energy above $3 \mathrm{EeV}$ are recorded with nearly $100 \%$ efficiency over the area covered by the surface array. The nonuniformity of the exposure in right ascension is below $1 \%$, negligible in the context of the present analysis. The dependence of the exposure on declination is calculated from the latitude of the detector and the full acceptance for showers up to $60^{\circ}$ zenith angle.

A key element of our study is the probability $P$ for a set of $N$ events from an isotropic flux to contain $k$ or more events at a maximum angular distance $\psi$ from any member of a collection of candidate point sources. $\mathbf{P}$ is given by the cumulative binomial distribution $\sum_{j=k}^{N} C_{j}^{N} p^{j}(1-$ $p)^{N-j}$, where the parameter $p$ is the fraction of the sky (weighted by the exposure) defined by the regions at angular separation less than $\psi$ from the selected sources.

We analyze the degree of correlation of our data with the directions of AGN referenced in the $\mathrm{V}-\mathrm{C}$ catalog $(12)$. This catalog does not contain all existing AGN and is not an unbiased statistical sample of them. This is not an obstacle to demonstrating the existence of anisotropies but may affect our ability to identify the cosmic-ray sources unambiguously. The catalog contains 694 active galaxies with redshifts $z \leq 0.024$, corresponding to distances $D$ smaller than $100 \mathrm{Mpc}$ (19). At larger distances, and around the Galactic plane, the catalog is increasingly incomplete. 
Exploration and confirmation Using data acquired between 1 January 2004 and 26 May 2006, we scanned for the minimum of $P$ in the three-dimensional parameter space defined by maximum angular separations $\psi$, maximum redshifts $z_{\max }$, and energy thresholds $E_{t h}$. The lower limit for the scan in $\psi$ corresponds to the angular resolution of the surface array. Our scan in energy threshold and maximum distance was motivated by the assumption that cosmic rays with the highest energies are the ones that are least deflected by intervening magnetic fields and that have the smallest probability of arrival from very distant sources due to the GZK effect (3, 4$)$.

We found a minimum of $P$ for the parameters $\psi=3.1^{\circ}, z_{\max }=0.018\left(D_{\max }=75 \mathrm{Mpc}\right)$, and $E_{t h}=56 \mathrm{EeV}$. For these values, 12 events among 15 correlate with the selected AGN, whereas only 3.2 were expected by chance if the flux were isotropic. This observation motivated the definition of a test to validate the result with an independent data set, with parameters specified a priori, as is required by the Auger source and anisotropy search methodology (20,21).

The Auger search protocol was designed as a sequence of tests to be applied after the observation of each new event with energy above $56 \mathrm{EeV}$. The total probability of incorrectly rejecting the isotropy hypothesis along the sequence was set to a maximum of $1 \%$. The parameters for the prescribed test were chosen as those, given above, that led to the minimum of $P$ in the exploratory scan. The probability of a chance correlation at the chosen angular scale of a single cosmic ray with the selected astronomical objects is $p=0.21$ if the flux were isotropic. The test was applied to data collected between 27 May 2006 and 31 August 2007, with exactly the same reconstruction algorithms, energy calibration, and quality cuts for event selection as in the exploratory scan. In these independent data, there are 13 events with energy above $56 \mathrm{EeV}$, of which 8 have arrival directions closer than $3.1^{\circ}$ from the positions of AGN less than $75 \mathrm{Mpc}$ away, with 2.7 expected on average. The probability that this configuration would occur by chance if the flux were isotropic is $1.7 \times 10^{-3}$. Following our search protocol and based on the 
independent data set alone, we reject the hypothesis of isotropy in the distribution of the arrival directions of cosmic rays with the highest energies with at least a $99 \%$ confidence level.

Results Having determined that an anisotropy exists, based on the a priori prescription, we rescanned the full data set from 1 January 2004 to 31 August 2007 using the method described above to substantiate the observed correlation. We used steps of $0.1^{\circ}$ in $\psi$, in the range $1^{\circ} \leq$ $\psi \leq 8^{\circ}$, and 0.001 in $z_{\max }$, in the range $0 \leq z_{\max } \leq 0.024$. We also used a newer version of our reconstruction and calibration algorithm that gives slightly different reconstructed directions and energies. These small differences, well within our reconstruction uncertainty, modify the final event selection, but this has minor consequences on the value of the parameters $\psi, z_{\max }$, and $E_{t h}$ that maximize the correlation signal. We start the scan with the event of highest energy and add events one by one in order of decreasing energy, down to $E_{t h}=40 \mathrm{EeV}$.

Strong correlation signals occur for energy thresholds around $60 \mathrm{EeV}$ and several combinations of the other parameters in the range $\psi \leq 6^{\circ}$, and $z_{\max } \leq 0.024\left(D_{\max }<100 \mathrm{Mpc}\right)$. The absolute minimum value of $P$ occurs for the 27 events with the highest energies (above $57 \mathrm{EeV}$ in the new analysis). We generated simulated sets of directions, drawn from an isotropic distribution in proportion to the relative exposure of the observatory. Performing an identical scan on those simulated samples to that applied to the real data, we obtain smaller or equal values of $P$ in $\sim 10^{-5}$ of the simulated direction sets.

We present (Figure 2) a sky map in Galactic coordinates of our 27 highest-energy events ( $E>57 \mathrm{EeV}$ ), as determined by our most recent version of the reconstruction code. The anisotropy is clearly visible. We note the proximity of several events close to the supergalactic plane, and also that two events arrive within $3^{\circ}$ of Centaurus A, one of the closest AGN, marked in white on the figure. 
Discussion With the statistics of our present data set, the observed correlation is significant for maximum distances to $\mathrm{AGN}$ of up to $100 \mathrm{Mpc}$, for maximum angular separations of up to $6^{\circ}$, and for energy thresholds around $60 \mathrm{EeV}$. Those numbers are to be taken as indicative because the minimization of $\mathbf{P}$ is not totally exempt from biases. Accidental correlation with foreground AGN different from the actual sources may induce bias towards smaller maximum source distances while accidental correlation with distant background ones may reduce the optimal maximum angular separation by a few degrees.

Under the simplifying assumptions of a uniform distribution of sources with equal intrinsic luminosity and continuous energy loss in the cosmic microwave background due to the GZK effect (3. 4), 90\% of the protons arriving at Earth with energy exceeding $60 \mathrm{EeV}$ originate from sources closer than $200 \mathrm{Mpc}$. This (somewhat arbitrarily defined) "GZK horizon" decreases rapidly with increasing energy and drops to $90 \mathrm{Mpc}$ for energies exceeding $80 \mathrm{EeV}$. The relation between the horizon distance and the value of $D_{\max }$ that minimizes $\mathrm{P}$ is not a simple one, given the possible biases in the method, which has nonuniform sensitivity over the range of parameters scanned. Increasing catalog incompleteness also prevents confidently scanning over sources at distances much larger than $100 \mathrm{Mpc}$. Moreover, the local density and luminosities of sources could have significant departures from the uniformity assumed in the GZK horizon scale for a given energy threshold. Taking into consideration these caveats, in addition to the uncertainty in the reconstructed energies, the range of $D_{\max }$ and $E_{t h}$ over which we observe a significant correlation is compatible with the frequently made assumption that the highest energy cosmic rays are protons experiencing predicted GZK energy losses. We note that the correlation increases abruptly at the energy threshold of $57 \mathrm{EeV}$, which coincides with the point on the energy spectrum recently reported from the observatory at which the flux is reduced by $\sim 50 \%$ with respect to a power law extrapolation of lower-energy observations (17).

If the regular component of the galactic magnetic field is coherent over scales of $1 \mathrm{kpc}$ with a 
strength of a few $\mu \mathrm{G}$, as indicated by data from studies of pulsars $(22)$, the observed correlation over an angular scale of only a few degrees for $E \sim 60 \mathrm{EeV}$ is indicative that most of the primaries are not heavy nuclei.

These features are compatible with the interpretation that the correlation we observe is evidence for the GZK effect and the hypothesis that the highest-energy cosmic rays reaching Earth are mostly protons from nearby sources.

The catalog of AGN that we use is increasingly incomplete near the Galactic plane, where extinction from dust in the Milky Way reduces the sensitivity of observations. Deflections from the Galactic magnetic field are also expected to be significantly larger than average for cosmic rays that arrive at equatorial Galactic latitudes, because they traverse a longer distance across any regular Galactic magnetic component. These effects are likely to have some impact upon the estimate of the strength of the correlation. Six out of the eight events that do not correlate with AGN positions within our prescribed parameters and reconstruction code lie less than $12^{\circ}$ away from the Galactic plane.

Despite its strength, the correlation that we observe with nearby AGN from the V-C cata$\log$ cannot be used alone as a proof that AGN are the sources. Other sources, as long as their distribution within the GZK horizon is sufficiently similar to that of the AGN, could lead to a significant correlation between the arrival directions of cosmic rays and the AGN positions. Such correlations are under investigation in particular for the Infra-Red Astronomical Satellite (IRAS) galaxies. The autocorrelation signal of the highest-energy events is also being investigated. It shows departures from isotropic expectations at angular scales between $5^{\circ}$ and $20^{\circ}(23)$ and serves as an additional tool to identify the spatial distribution of the sources.

Conclusion We have demonstrated the anisotropy of the arrival directions of the highestenergy cosmic rays and their extragalactic origin. Our observations are consistent with the 
hypothesis that the rapid decrease of flux measured by the Pierre Auger Observatory above $60 \mathrm{EeV}$ is due to the GZK effect and that most of the cosmic rays reaching Earth in that energy range are protons from nearby astrophysical sources, either AGN or other objects with a similar spatial distribution.

The number of high-energy cosmic-ray events recorded so far by the Pierre Auger Observatory and analysed in this work corresponds to 1.2 years of operation of the complete southern array. The data set that the observatory will gather in just a few more years should offer a better chance to unambiguously identify the sources. The pattern of correlations of cosmic-ray events with their sources could also assist in determining the properties of the intervening magneticfield structures and in particle physics explorations at the largest energies. Astronomy based on cosmic rays with the highest energies opens a new window on the nearby universe.

\section{References and Notes}

1. J. Linsley, Phys. Rev. Lett. 10, 146 (1963).

2. J. Abraham et al. [Pierre Auger Collaboration], Nucl. Instr. and Meth., A 523, 50 (2004).

3. K. Greisen, Phys. Rev Lett. 16, 748 (1966).

4. G.T. Zatsepin and V.A. Kuzmin, Sov. Phys. JETP. Lett. 4, 78 (1966).

5. V. S. Berezinsky and S. I. Grigorieva, Astron. \& Astrophys. 199, 1 (1988).

6. N. Hayashida et al., Phys. Rev. Lett. 77, 1000 (1996).

7. R. U. Abbasi et al [The HiRes collaboration] Astrophys. J. 610, L73 (2004).

8. T. Stanev, P.L. Biermann, J. Lloyd-Evans, J.P. Rachen and A.A. Watson, Phys. Rev. Lett. 75, 3056 (1995). 
9. Y. Uchihori, M. Nagano, M. Takeda, M. Teshima, J. Lloyd-Evans and A.A. Watson, Astropart. Phys. 13, 151 (2000).

10. V. L. Ginzburg and S. I. Syrovatskii, The Origin of Cosmic Rays, Pergamon Press (Oxford, 1964).

11. A.M. Hillas, Annu. Rev. Astron. Astrophys. 22, 425 (1984).

12. M.-P. Véron-Cetty and P. Véron, Astron. \& Astrophys. 455, 773 (2006). We acknowledge use of the VizieR catalogue access tool, CDS, Strasbourg, France, at http://vizier.ustrasbg.fr/viz-bin/VizieR

13. D. Allard et al. [Pierre Auger Collaboration], Proceedings of the 29th International Cosmic Ray Conference, (2005) Pune, India 7, 287. [astro-ph/0511104]

14. C. Bonifazi, A. Letessier-Selvon and E. M. Santos, Astropart. Phys. (in press). arXiv:0705.1856 [astro-ph]

15. C. Bonifazi [Pierre Auger Collaboration], Proceedings of the 29th International Cosmic Ray Conference (2005) Pune, India 7, 17.

16. M. Ave [Pierre Auger Collaboration], Proceedings of the 30th International Cosmic Ray Conference, (2007) Merida, Mexico. arXiv:0709.2125 [astro-ph].

17. M. Roth [Pierre Auger Collaboration], Proceedings of the 30th International Cosmic Ray Conference, (2007) Merida, Mexico. arXiv:0706.2096 [astro-ph].

18. B.R. Dawson [Pierre Auger Collaboration], Proceedings of the 30th International Cosmic Ray Conference, (2007) Merida, Mexico. arXiv:0706.1105 [astro-ph]. 
19. A redshift $z$ corresponds to a distance $42 \mathrm{Mpc} \times(z / 0.01)$ for a Hubble constant $H_{0}=$ $71 \mathrm{~km} \mathrm{~s}^{-1} \mathrm{Mpc}^{-1}$.

20. R.W. Clay [Pierre Auger Collaboration], Proceedings of the 28th International Cosmic Ray Conference (2003) Tsukuba, Japan 1, 421.

21. B. Revenu [Pierre Auger Collaboration], Proceedings of the 29th International Cosmic Ray Conference (2005) Pune, India 7, 75.

22. J. L. Han, R. N. Manchester, A. G. Lyne, G. J. Qiao, and W. van Straten, Astrophys. J. 642, 868 (2006).

23. S. Mollerach [Pierre Auger Collaboration], Proceedings of the 30th International Cosmic Ray Conference, (2007) Merida, Mexico. arXiv:0706.1749 [astro-ph]

24. We are grateful to the following agencies and organizations for financial support: Gobierno De La Provincia de Mendoza, Comisión Nacional de Energía Atómica, Municipalidad de Malargüe, Fundación Antorchas, Argentina; the Australian Research Council; Conselho Nacional de Desenvolvimento Científico e Tecnológico (CNPq), Financiadora de Estudos e Projetos do Ministerio da Ciencia e Tecnologia (FINEP / MCT), Fundação de Amparo à Pesquisa do Estado de Rio de Janeiro (FAPERJ), Fundação de Amparo à Pesquisa do Estado de São Paulo (FAPESP), Brazil; Ministry of Education, Youth and Sports of the Czech Republic; Centre National de la Recherche Scientifique (CNRS), Conseil Régional Ile-deFrance, Département Physique Nucléaire et Corpusculaire (PNC-IN2P3/CNRS), Département Sciences de l'Univers (SDU-INSU/CNRS), France; Bundesministerium für Bildung und Forschung (BMBF), Deutsche Forschungsgemeinschaft (DFG), Finanzministerium BadenWürttemberg, Helmholtz-Gemeinschaft Deutscher Forschungszentren (HGF), Ministerium für Wissenschaft und Forschung, Nordrhein Westfalen, Ministerium für Wissenschaft, Forschung 
und Kunst, Baden-Württemberg, Germany; Istituto Nazionale di Fisica Nucleare (INFN), Ministero dell'Istruzione, dell'Università e della Ricerca (MIUR), Italy; Consejo Nacional de Ciencia y Tecnología (CONACYT), Mexico; Ministerie van Onderwijs, Cultuur en Wetenschap, Nederlandse Organisatie voor Wetenschappelijk Onderzoek (NWO), Stichting voor Fundamenteel Onderzoek der Materie (FOM), Netherlands; Ministry of Science and Higher Education, Poland; Fundação para a Ciência e a Tecnologia, Portugal; Ministry for Higher Education, Science, and Technology, Slovenian Research Agency, Slovenia; Comunidad de Madrid, Consejería de Educacíon de la Comunidad de Castilla La Mancha, FEDER funds, Ministerio de Educacíon y Ciencia, Xunta de Galicia, Spain; Science and Technology Facilities Council, United Kingdom; Department of Energy, National Science Foundation, The Grainger Foundation, USA; América Latina Formación Académica - European Community / High Energy physics Latin-american European Network (ALFA-EC / HELEN), and UNESCO. 


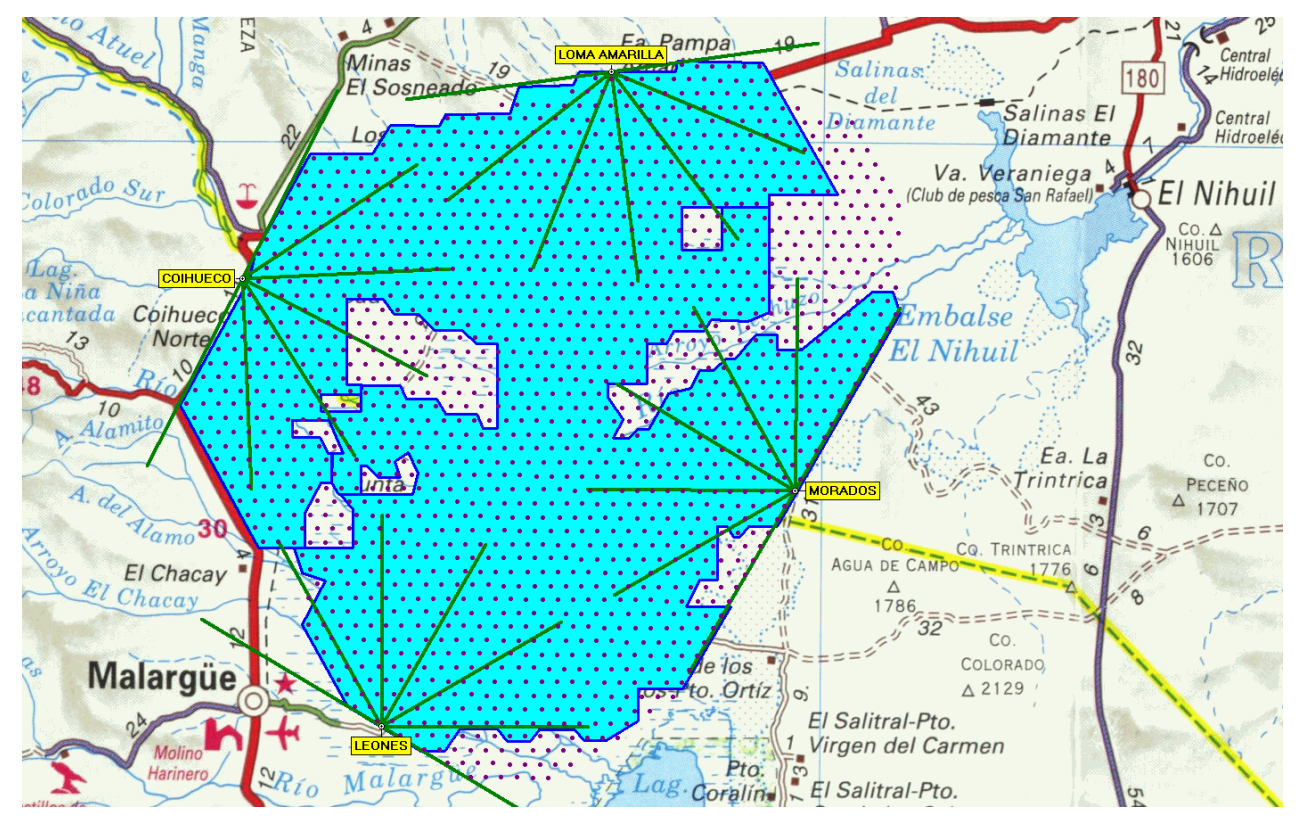

Figure 1: Layout of the Pierre Auger Southern Observatory. The dots represent the position of each of the 1600 SD stations. The 1430 SD stations deployed and activated as of 30 September 2007 lie in the area shaded blue. The 4 FD sites are labeled in yellow, with green lines indicating the field of view of the six telescopes at each site. To give the scale of the Observatory, the lengths of the green line correspond to $20 \mathrm{~km}$. 


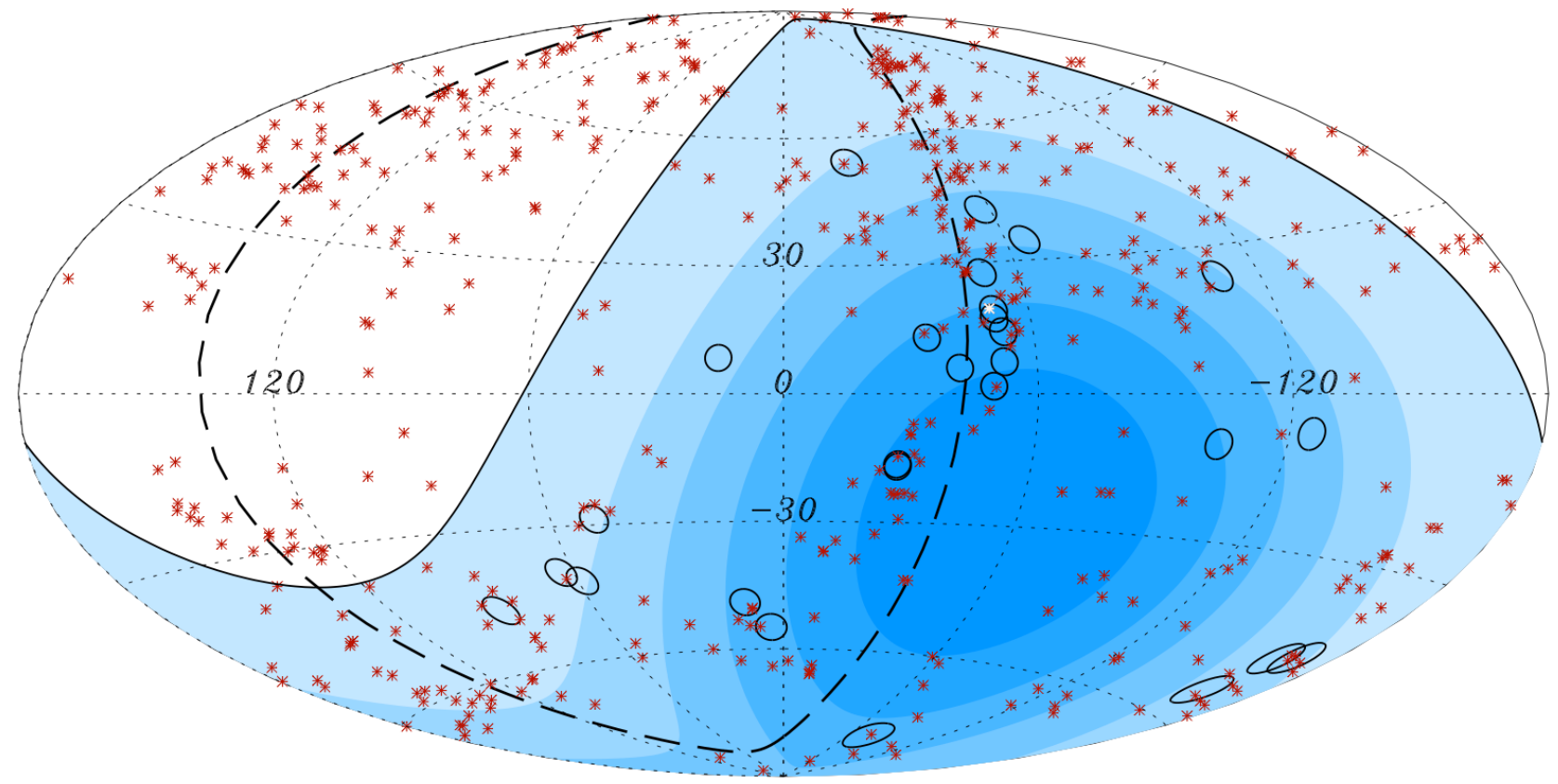

Figure 2: Aitoff projection of the celestial sphere in galactic coordinates with circles of radius $3.1^{\circ}$ centered at the arrival directions of the 27 cosmic rays with highest energy detected by the Pierre Auger Observatory. The positions of the 472 AGN (318 in the field of view of the Observatory) with redshift $z \leq 0.018$ ( $D<75 \mathrm{Mpc}$ ) from the $12^{\text {th }}$ edition of the catalog of quasars and active nuclei $(12)$ are indicated by red asterisks. The solid line represents the border of the field of view (zenith angles smaller than $60^{\circ}$ ). Darker color indicates larger relative exposure. Each colored band has equal integrated exposure. The dashed line is the supergalactic plane. Centaurus A, one of our closest AGN, is marked in white. 


\section{The Pierre Auger Collaboration}

J. Abraham ${ }^{6}$, P. Abreu ${ }^{59}$, M. Aglietta ${ }^{45}$, C. Aguirre $^{8}$, D. Allard ${ }^{24}$, I. Allekotte ${ }^{1}$, J. Allen ${ }^{78}$, P. Allison ${ }^{80}$, C. Alvarez ${ }^{49}$, J. Alvarez-Muñiz ${ }^{65}$, M. Ambrosio ${ }^{48}$, L. Anchordoqui ${ }^{93,79}$, S. Andringa ${ }^{59}$,

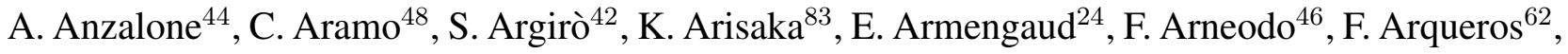
T. $\operatorname{Asch}^{30}$, H. Asorey ${ }^{1}$, P. Assis ${ }^{59}$, B.S. Atulugama ${ }^{81}$, J. Aublin ${ }^{26}$, M. Ave ${ }^{84}$, G. Avila ${ }^{4,6}$, T. Bäcker ${ }^{34}$, D. Badagnani ${ }^{5}$, A.F. Barbosa ${ }^{10}$, D. Barnhill ${ }^{83}$, S.L.C. Barroso ${ }^{16}$, P. Bauleo ${ }^{72}$, J. Beatty ${ }^{80}$, T. Beau ${ }^{24}$, B.R. Becker ${ }^{89}$, K.H. Becker ${ }^{28}$, J.A. Bellido ${ }^{81}$, S. BenZvi ${ }^{92,74}$, C. Berat $^{27}$, T. Bergmann ${ }^{33}$, P. Bernardini ${ }^{43}$, X. Bertou ${ }^{1}$, P.L. Biermann ${ }^{31}$, P. Billoir ${ }^{26}$, O. Blanch-Bigas ${ }^{26}$, F. Blanco ${ }^{62}$, P. Blasi ${ }^{75,37,47}$, C. Bleve ${ }^{68}$, H. Blümer ${ }^{33,29}$, M. Boháčováá 22 C. Bonifazii ${ }^{26,10}$, R. Bonino ${ }^{45}$, M. Boratav ${ }^{26}$, J. Brack ${ }^{72,85}$, P. Brogueira ${ }^{59}$, W.C. Brown ${ }^{73}$, P. Buchholz ${ }^{34}$, A. Bueno ${ }^{64}$, R.E. Burton ${ }^{70}$, N.G. Busca ${ }^{24}$, K.S. Caballero-Mora ${ }^{33}$, B. Cai ${ }^{87}$, D.V. Camin ${ }^{38}$, L. Caramete ${ }^{31}$, R. Caruso ${ }^{41}$, W. Carvalho ${ }^{12}$, A. Castellina ${ }^{45}$, O. Catalano ${ }^{44}$, G. Cataldi ${ }^{43}$, L. Cazón-Boado ${ }^{84}$, R. Cester ${ }^{42}$, J. Chauvin ${ }^{27}$, A. Chiavassa ${ }^{45}$, J.A. Chinellato ${ }^{14}$, A. Chou ${ }^{78,75}$, J. Chye ${ }^{77}$, P.D.J. Clark ${ }^{67}$, R.W. Clay ${ }^{7}$, E. Colombo ${ }^{3}$, R. Conceição ${ }^{59}$, B. Connolly ${ }^{90,74}$, F. Contreras ${ }^{4}$, J. Coppens ${ }^{53,55}$,

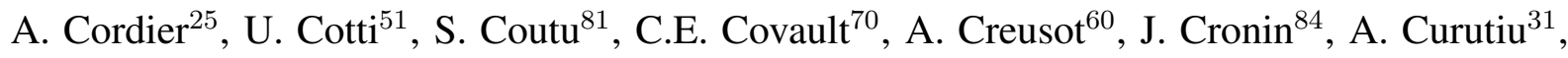
S. Dagoret-Campagne ${ }^{25}$, K. Daumiller ${ }^{29}$, B.R. Dawson ${ }^{7}$, R.M. de Almeida ${ }^{14}$, C. De Donato ${ }^{38}$, S.J. de Jong ${ }^{53}$, G. De La Vega ${ }^{6}$, W.J.M. de Mello Junior ${ }^{14}$, J.R.T. de Mello Neto ${ }^{84,19}$, I. De Mitri $^{43}$, V. de Souza $^{33}$, L. del Peral ${ }^{63}$, O. Deligny ${ }^{23}$, A. Della Selva ${ }^{39}$, C. Delle Fratte ${ }^{40}$, H. Dembinski ${ }^{32}$, C. Di Giulio ${ }^{40}$, J.C. Diaz ${ }^{77}$, C. Dobrigkeit ${ }^{14}$, J.C. D’Olivo ${ }^{52}$, D. Dornic ${ }^{23}$, A. Dorofeev ${ }^{76}$,

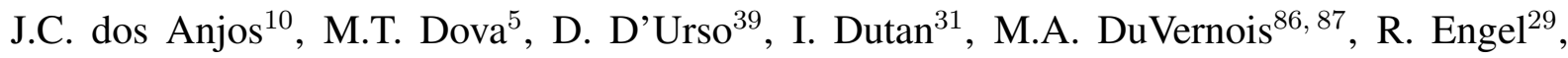
L. Epele ${ }^{5}$, M. Erdmann ${ }^{32}$, C.O. Escobar ${ }^{14}$, A. Etchegoyen ${ }^{3}$, P. Facal San Luis ${ }^{65}$, H. Falcke ${ }^{53,56}$, G. Farrar $^{78}$, A.C. Fauth ${ }^{14}$, N. Fazzini ${ }^{75}$, A. Fernández ${ }^{49}$, F. Ferrer ${ }^{70}$, S. Ferry ${ }^{60}$, B. Fick ${ }^{77}$, A. Filevich ${ }^{3}$, A. Filipčič ${ }^{60}$, I. Fleck $^{34}$, R. Fonte ${ }^{41}$, C.E. Fracchiolla ${ }^{11}$, W. Fulgione ${ }^{45}$, B. García ${ }^{6}$, D. García Gámez ${ }^{64}$, D. Garcia-Pinto ${ }^{62}$, X. Garrido ${ }^{25}$, H. Geenen ${ }^{28}$, G. Gelmini $^{83}$, H. Gemmeke ${ }^{30}$, P.L. Ghia ${ }^{23,45}$, M. Giller ${ }^{58}$, H. Glass ${ }^{75}$, M.S. Gold ${ }^{89}$, G. Golup ${ }^{1}$, F. Gomez Albarracin ${ }^{5}$, M. Gómez 
Berisso $^{1}$, R. Gómez Herrero ${ }^{63}$, P. Gonçalves ${ }^{59}$, M. Gonçalves do Amaral ${ }^{20}$, D. Gonzalez ${ }^{33}$, J.G. Gonzalez ${ }^{76}$, M. González ${ }^{50}$, D. Góra ${ }^{33,57}$, A. Gorgi ${ }^{45}$, P. Gouffon ${ }^{12}$, V. Grassi ${ }^{38}$, A.F. Grillo ${ }^{46}$, C. Grunfeld ${ }^{5}$, Y. Guardincerri ${ }^{5}$, F. Guarino ${ }^{39}$, G.P. Guedes ${ }^{15}$, J. Gutiérrez ${ }^{63}$, J.D. Hague ${ }^{89}$, J.C. Hamilton ${ }^{24}$, P. Hansen ${ }^{65}$, D. Harari ${ }^{1}$, S. Harmsma ${ }^{54}$, J.L. Harton ${ }^{23,72}$, A. Haungs ${ }^{29}$, T. Hauschildt ${ }^{45}$, M.D. Healy ${ }^{83}$, T. Hebbeker ${ }^{32}$, D. Heck ${ }^{29}$, C. Hojvat ${ }^{75}$, V.C. Holmes ${ }^{7}$, P. Homola ${ }^{57}$, J. Hörandel ${ }^{53}$, A. Horneffer ${ }^{53}$, M. Horvat ${ }^{60}$, M. Hrabovský ${ }^{22}$, T. Huege ${ }^{29}$, M. Iarlori ${ }^{37}$, A. Insolia ${ }^{41}$, F. Ionita ${ }^{84}$, A. Italiano ${ }^{41}$, M. Kaducak ${ }^{75}$, K.H. Kampert ${ }^{28}$, T. Karova ${ }^{22}$, B. Kégl ${ }^{25}$, B. Keilhauer ${ }^{33}$, E. Kemp ${ }^{14}$, R.M. Kieckhafer ${ }^{77}$, H.O. Klages ${ }^{29}$, M. Kleifges ${ }^{30}$, J. Kleinfeller ${ }^{29}$, R. Knapik ${ }^{72}$, J. Knapp ${ }^{68}$, D.H. Koang ${ }^{27}$, A. Kopmann ${ }^{30}$, A. Krieger ${ }^{3}$, O. Krömer ${ }^{30}$, D. Kümpel ${ }^{28}$, N. Kunka ${ }^{30}$, A. Kusenko ${ }^{83}$, G. La Rosa ${ }^{44}$, C. Lachaud $^{24}$, B.L. Lago ${ }^{19}$, D. Lebrun ${ }^{27}$, P. LeBrun ${ }^{75}$, J. Lee ${ }^{83}$, M.A. Leigui de Oliveira ${ }^{18}$, A. Letessier-Selvon ${ }^{26}$, M. Leuthold ${ }^{32}$, I. Lhenry-Yvon ${ }^{23}$, R. López ${ }^{49}$, A. Lopez Agüera $^{65}$, J. Lozano Bahilo ${ }^{64}$, M.C. Maccarone ${ }^{44}$, C. Macolino ${ }^{37}$, S. Maldera ${ }^{45}$, M. Malek ${ }^{75}$, G. Mancarella ${ }^{43}$, M.E. Manceñido ${ }^{5}$, D. Mandat ${ }^{22}$, P. Mantsch ${ }^{75}$, A.G. Mariazzi ${ }^{5}$, I.C. Maris ${ }^{33}$, D. Martello ${ }^{43}$, J. Martínez ${ }^{50}$, O. Martínez Bravo ${ }^{49}$, H.J. Mathes ${ }^{29}$, J. Matthews ${ }^{76,82}$, J.A.J. Matthews ${ }^{89}$, G. Matthiae ${ }^{40}$, D. Maurizio ${ }^{42}$, P.O. Mazur ${ }^{75}$, T. $\mathrm{McCauley}^{79}$, M. $\mathrm{McEwen}^{76}$, R.R. McNeil ${ }^{76}$, M.C. Medina ${ }^{3}$, G. Medina-Tanco ${ }^{52}$, A. Meli ${ }^{31}$, D. Melo ${ }^{3}$, E. Menichetti ${ }^{42}$, A. Menschikov ${ }^{30}$, Chr. Meurer ${ }^{29}$, R. Meyhandan ${ }^{54}$, M.I. Micheletti ${ }^{3}$, G. Miele ${ }^{39}$, W. Miller ${ }^{89}$, S. Mollerach ${ }^{1}$, M. Monasor ${ }^{62,63}$, D. Monnier Ragaigne ${ }^{25}$, F. Montanet ${ }^{27}$, B. Morales ${ }^{52}$, C. Morello ${ }^{45}$, E. Moreno ${ }^{49}$, J.C. Moreno ${ }^{5}$, C. Morris ${ }^{80}$, M. Mostafáa ${ }^{91}$, M.A. Muller ${ }^{14}$, R. Mussa ${ }^{42}$, G. Navarra ${ }^{45}$, J.L. Navarro ${ }^{64}$, S. Navas ${ }^{64}$, P. Necesal ${ }^{22}$, L. Nellen ${ }^{52}$, C. Newman-Holmes ${ }^{75}$, D. Newton ${ }^{68,65}$, T. Nguyen Thi ${ }^{94}$, N. Nierstenhöfer ${ }^{28}$, D. Nitz ${ }^{77}$, D. Nosek ${ }^{21}$, L. Nožka ${ }^{22}$, J. Oehlschläger ${ }^{29}$, T. Ohnuki ${ }^{83}$, A. Olinto ${ }^{24,84}$, V.M. Olmos-Gilbaja ${ }^{65}$, M. Ortiz ${ }^{62}$, S. Ostapchenko ${ }^{33}$, L. Otero ${ }^{6}$, D. Pakk Selmi-Dei ${ }^{14}$, M. Palatka ${ }^{22}$, J. Pallotta ${ }^{6}$, G. Parente ${ }^{65}$, E. Parizot ${ }^{24}$, S. Parlati ${ }^{46}$, S. Pastor ${ }^{61}$, M. Patel ${ }^{68}$, T. Paul ${ }^{79}$, V. Pavlidou ${ }^{84}$, K. Payet $^{27}$, M. Pech ${ }^{22}$, J. Pȩkala ${ }^{57}$, R. Pelayo ${ }^{50}$, I.M. Pepe ${ }^{17}$, L. Perrone ${ }^{43}$, S. Petrera ${ }^{37}$, P. Petrinca ${ }^{40}$, Y. Petrov ${ }^{72}$, Diep Pham Ngoc ${ }^{94}$, Dong Pham Ngoc ${ }^{94}$, T.N. Pham Thi ${ }^{94}$, A. Pichel ${ }^{2}$, R. Piegaia ${ }^{5}$, 
T. Pierog ${ }^{29}$, M. Pimenta ${ }^{59}$, T. Pinto ${ }^{61}$, V. Pirronello ${ }^{41}$, O. Pisanti ${ }^{39}$, M. Platino ${ }^{3}$, J. Pochon ${ }^{1}$, T.A. Porter ${ }^{76}$, P. Privitera ${ }^{40}$, M. Prouza ${ }^{22,74}$, E.J. Quel ${ }^{6}$, J. Rautenberg ${ }^{28}$, S. Reucroft ${ }^{79}$, B. Revenu ${ }^{24}$, F.A.S. Rezende ${ }^{10}$, J. Řídký2 ${ }^{22}$, S. Riggi ${ }^{41}$, M. Risse ${ }^{28}$, C. Rivière ${ }^{27}$, V. Rizi ${ }^{37}$, M. Roberts ${ }^{81}$, C. Robledo ${ }^{49}$, G. Rodriguez ${ }^{65}$, D. Rodríguez Frías ${ }^{63}$, J. Rodriguez Martino ${ }^{40}$, J. Rodriguez Rojo $^{4}$, I. Rodriguez-Cabo ${ }^{65}$, G. $\operatorname{Ros}^{62,63}$, J. Rosado ${ }^{62}$, M. Roth ${ }^{29}$, B. Rouillé-d'Orfeuil ${ }^{24}$, E. Roulet ${ }^{1}$, A.C. Rovero ${ }^{2}$, F. Salamida ${ }^{37}$, H. Salazar ${ }^{49}$, G. Salina ${ }^{40}$, F. Sánchez ${ }^{52}$, M. Santander ${ }^{4}$, C.E. Santo ${ }^{59}$, E.M. Santos ${ }^{26,10}$, F. Sarazin ${ }^{71}$, S. Sarkar ${ }^{66}$, R. Sato ${ }^{4}$, V. Scherini ${ }^{28}$, H. Schieler ${ }^{29}$, F. Schmidt ${ }^{84}$, T. Schmidt ${ }^{33}$, O. Scholten ${ }^{54}$, P. Schovánek ${ }^{22}$, F. Schüssler ${ }^{29}$, S.J. Sciutto ${ }^{5}$, M. Scuderi ${ }^{41}$, A. Segreto ${ }^{44}$, D. Semikoz ${ }^{24}$, M. Settimo ${ }^{43}$, R.C. Shellard ${ }^{10,11}$, I. Sidelnik ${ }^{3}$, B.B. Siffert ${ }^{19}$, G. Sigl ${ }^{24}$, N. Smetniansky De Grande ${ }^{3}$, A. Smiałkowski ${ }^{58}$, R. Šmída ${ }^{22}$, A.G.K. Smith ${ }^{7}$, B.E. Smith ${ }^{68}$, G.R. Snow ${ }^{88}$, P. Sokolsky ${ }^{91}$, P. Sommers ${ }^{81}$, J. Sorokin ${ }^{7}$, H. Spinka ${ }^{69,75}$, R. Squartini ${ }^{4}$, E. Strazzeri ${ }^{40}$, A. Stutz ${ }^{27}$, F. Suarez ${ }^{45}$, T. Suomijärvii ${ }^{23}$, A.D. Supanitsky ${ }^{52}$, M.S. Sutherland ${ }^{80}$, J. Swain ${ }^{79}$, Z. Szadkowski ${ }^{58}$,

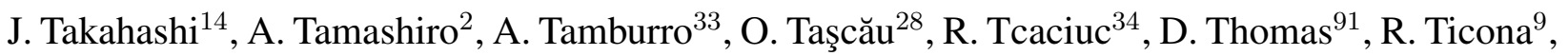
J. Tiffenberg ${ }^{5}$, C. Timmermans ${ }^{55,53}$, W. Tkaczyk ${ }^{58}$, C.J. Todero Peixoto ${ }^{14}$, B. Tomé ${ }^{59}$, A. Tonachini ${ }^{42}$, D. Torresi ${ }^{44}$, P. Travnicek ${ }^{22}$, A. Tripathi ${ }^{83}$, G. Tristram ${ }^{24}$, D. Tscherniakhovski ${ }^{30}$, M. Tueros $^{5}$, V. Tunnicliffe ${ }^{67}$, R. Ulrich ${ }^{29}$, M. Unger ${ }^{29}$, M. Urban ${ }^{25}$, J.F. Valdés Galicia ${ }^{52}$, I. Valiño ${ }^{65}$, L. Valore ${ }^{39}$, A.M. van den Berg ${ }^{54}$, V. van Elewyck ${ }^{23}$, R.A. Vázquez ${ }^{65}$, D. Veberič ${ }^{60}$, A. Veiga ${ }^{5}$, A. Velarde ${ }^{9}$, T. Venters ${ }^{84,24}$, V. Verzi ${ }^{40}$, M. Videla ${ }^{6}$, L. Villaseñor ${ }^{51}$, S. Vorobiov ${ }^{60}$, L. Voyvodic $^{75}$, H. Wahlberg ${ }^{5}$, O. Wainberg ${ }^{3}$, P. Walker ${ }^{67}$, D. Warner ${ }^{72}$, A.A. Watson ${ }^{68}$, S. Westerhoff ${ }^{92}$, G. Wieczorek ${ }^{58}$, L. Wiencke ${ }^{71}$, B. Wilczyńska ${ }^{57}$, H. Wilczyński ${ }^{57}$, C. Wileman ${ }^{68}$, M.G. Winnick ${ }^{7}, \mathrm{H} \mathrm{Wu}^{25}$, B. Wundheiler ${ }^{3}$, J. Xu ${ }^{30}$, T. Yamamoto ${ }^{84}$, P. Younk ${ }^{91}$, E. Zas ${ }^{65}$, D. Zavrtanik ${ }^{60}$, M. Zavrtanik ${ }^{60}$, A. $Z_{e c h}{ }^{26}$, A. Zepeda ${ }^{50}$, M. Ziolkowski ${ }^{34}$

${ }^{1}$ Centro Atómico Bariloche (CNEA); Instituto Balseiro (UNCuyo), Río Negro, Argentina

${ }^{2}$ Instituto de Astronomía y Física del Espacio (CONICET), Buenos Aires, Argentina

${ }^{3}$ Centro Atómico Constituyentes, CNEA, Buenos Aires, Argentina 
${ }^{4}$ Pierre Auger Southern Observatory, Malargüe, Argentina

${ }^{5}$ Universidad Nacional de la Plata, IFLP/CONICET, La Plata, Argentina

${ }^{6}$ Universidad Tecnológica Nacional, Regionales Mendoza y San Rafael, Mendoza, Argentina

${ }^{7}$ University of Adelaide, Adelaide, S.A., Australia

${ }^{8}$ Universidad Catolica de Bolivia, La Paz, Bolivia

${ }^{9}$ Universidad Mayor de San Andrés, Bolivia

${ }^{10}$ Centro Brasileiro de Pesquisas Fisicas, Rio de Janeiro, RJ, Brazil

${ }^{11}$ Pontifícia Universidade Católica, Rio de Janeiro, RJ, Brazil

${ }^{12}$ Universidade de Sao Paulo, Inst. de Fisica, Sao Paulo, SP, Brazil

${ }^{14}$ Universidade Estadual de Campinas, IFGW, Campinas, SP, Brazil

${ }^{15}$ Univ. Estadual de Feira de Santana, Brazil

${ }^{16}$ Universidade Estadual do Sudoeste da Bahia, Vitoria da Conquista, BA, Brazil

${ }^{17}$ Universidade Federal da Bahia, Salvador, BA, Brazil

${ }^{18}$ Universidade Federal do ABC, Santo André, SP, Brazil

${ }^{19}$ Univ. Federal do Rio de Janeiro, Instituto de Física, Rio de Janeiro, RJ, Brazil

${ }^{20}$ Univ. Federal Fluminense, Inst. de Fisica, Niterói, RJ, Brazil

${ }^{21}$ Charles University, Institute of Particle \& Nuclear Physics, Prague, Czech Republic

${ }^{22}$ Institute of Physics of the Academy of Sciences of the Czech Republic, Prague, Czech Republic

${ }^{23}$ Institut de Physique Nucléaire, Université Paris-Sud, IN2P3/CNRS, Orsay, France

${ }^{24}$ Laboratoire AstroParticule et Cosmologie, Université Paris 7, IN2P3/CNRS, Paris, France

${ }^{25}$ Laboratoire de l'Accélérateur Linéaire, Université Paris-Sud, IN2P3/CNRS, Orsay, France

${ }^{26}$ Laboratoire de Physique Nucléaire et de Hautes Energies, Université Paris 6 \& 7, IN2P3/CNRS, Paris, France

${ }^{27}$ Laboratoire de Physique Subatomique et de Cosmologie, IN2P3/CNRS, Grenoble, France 
${ }^{28}$ Bergische Universität Wuppertal, Wuppertal, Germany

${ }^{29}$ Forschungszentrum Karlsruhe, Institut für Kernphysik, Karlsruhe, Germany

${ }^{30}$ Forschungszentrum Karlsruhe, Institut für Prozessdatenverarbeitung und Elektronik, Germany

${ }^{31}$ Max-Planck-Institut für Radioastronomie, Bonn, Germany

${ }^{32}$ RWTH Aachen University, III. Physikalisches Institut A, Aachen, Germany

${ }^{33}$ Universität Karlsruhe (TH), Institut für Experimentelle Kernphysik (IEKP), Karlsruhe, Germany

${ }^{34}$ Universität Siegen, Siegen, Germany

37 Università de l'Aquila and Sezione INFN, Aquila, Italy

${ }^{38}$ Università di Milano and Sezione INFN, Milan, Italy

${ }^{39}$ Università di Napoli "Federico II" and Sezione INFN, Napoli, Italy

${ }^{40}$ Università di Roma II "Tor Vergata" and Sezione INFN, Roma, Italy

${ }^{41}$ Università di Catania and Sezione INFN, Catania, Italy

${ }^{42}$ Università di Torino and Sezione INFN, Torino, Italy

${ }^{43}$ Università del Salento and Sezione INFN, Lecce, Italy

${ }^{44}$ Istituto di Astrofisica Spaziale e Fisica Cosmica di Palermo (INAF), Palermo, Italy

${ }^{45}$ Istituto di Fisica dello Spazio Interplanetario (INAF), Università di Torino and Sezione INFN, Torino, Italy

${ }^{46}$ INFN, Laboratori Nazionali del Gran Sasso, Assergi (L'Aquila), Italy

${ }^{47}$ Osservatorio Astrofisico di Arcetri, Florence, Italy

48 Sezione INFN di Napoli, Napoli, Italy

${ }^{49}$ Benemérita Universidad Autónoma de Puebla, Puebla, Mexico

${ }^{50}$ Centro de Investigación y de Estudios Avanzados del IPN (CINVESTAV), México, D.F., Mexico 
${ }^{51}$ Universidad Michoacana de San Nicolas de Hidalgo, Morelia, Michoacan, Mexico

${ }^{52}$ Universidad Nacional Autonoma de Mexico, Mexico, D.F., Mexico

${ }^{53}$ IMAPP, Radboud University, Nijmegen, Netherlands

${ }^{54}$ Kernfysisch Versneller Instituut, University of Groningen, Groningen, Netherlands

${ }^{55}$ NIKHEF, Amsterdam, Netherlands

56 ASTRON, Dwingeloo, Netherlands

${ }^{57}$ Institute of Nuclear Physics PAN, Krakow, Poland

${ }^{58}$ University of Łódź, Łódz, Poland

${ }^{59}$ LIP and Instituto Superior Técnico, Lisboa, Portugal

${ }^{60}$ Laboratory for Astroparticle Physics, University of Nova Gorica, Slovenia

${ }^{61}$ Instituto de Física Corpuscular, CSIC-Universitat de València, Valencia, Spain

${ }^{62}$ Universidad Complutense de Madrid, Madrid, Spain

${ }^{63}$ Universidad de Alcalá, Alcalá de Henares (Madrid), Spain

${ }^{64}$ Universidad de Granada \& C.A.F.P.E., Granada, Spain

${ }^{65}$ Universidad de Santiago de Compostela, Spain

${ }^{66}$ Rudolf Peierls Centre for Theoretical Physics, University of Oxford, Oxford, United Kingdom

${ }^{67}$ Institute of Integrated Information Systems, University of Leeds, United Kingdom

68 School of Physics and Astronomy, University of Leeds, United Kingdom

${ }^{69}$ Argonne National Laboratory, Argonne, IL, USA

${ }^{70}$ Case Western Reserve University, Cleveland, OH, USA

${ }^{71}$ Colorado School of Mines, Golden, CO, USA

${ }^{72}$ Colorado State University, Fort Collins, CO, USA

${ }^{73}$ Colorado State University, Pueblo, CO, USA

${ }^{74}$ Columbia University, New York, NY, USA 
${ }^{75}$ Fermilab, Batavia, IL, USA

${ }^{76}$ Louisiana State University, Baton Rouge, LA, USA

${ }^{77}$ Michigan Technological University, Houghton, MI, USA

${ }^{78}$ New York University, New York, NY, USA

${ }^{79}$ Northeastern University, Boston, MA, USA

${ }^{80}$ Ohio State University, Columbus, OH, USA

${ }^{81}$ Pennsylvania State University, University Park, PA, USA

${ }^{82}$ Southern University, Baton Rouge, LA, USA

${ }^{83}$ University of California, Los Angeles, CA, USA

${ }^{84}$ University of Chicago, Enrico Fermi Institute, Chicago, IL, USA

${ }^{85}$ University of Colorado, Boulder, CO, USA

${ }^{86}$ University of Hawaii, Honolulu, HI, USA

${ }^{87}$ University of Minnesota, Minneapolis, MN, USA

${ }^{88}$ University of Nebraska, Lincoln, NE, USA

${ }^{89}$ University of New Mexico, Albuquerque, NM, USA

${ }^{90}$ University of Pennsylvania, Philadelphia, PA, USA

${ }^{91}$ University of Utah, Salt Lake City, UT, USA

${ }^{92}$ University of Wisconsin, Madison, WI, USA

${ }^{93}$ University of Wisconsin, Milwaukee, WI, USA

${ }^{94}$ Institute for Nuclear Science and Technology, Hanoi, Vietnam 